
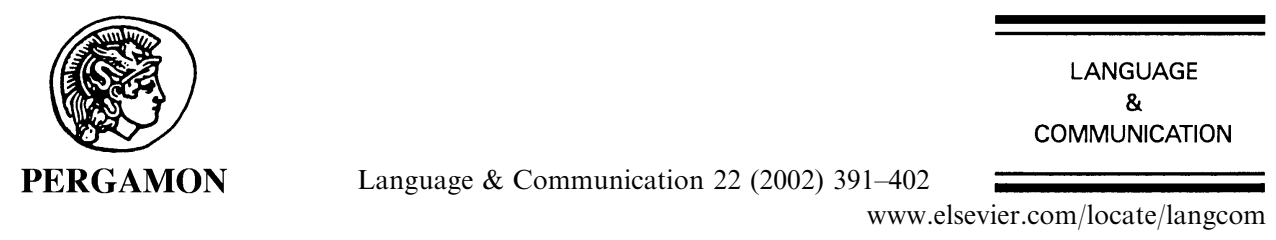

Editorial

\title{
Contexts of social action: guest editors' introduction
}

\author{
Context is one of those linguistic terms which is constantly used in all \\ kinds of context but never explained. (Asher, 1994, p. 731)
}

\section{Introduction}

In traditional linguistic accounts of context, one thinks of the immediate features of a speech situation, that is, a situation in which an expression is uttered. Thus, features such as time, location, speaker, hearer and preceding discourse are all parts of context. But context is a wider and more transcendental notion than what these accounts imply. For one thing, context is a relational concept relating social actions and their surroundings, relating social actions, relating individual actors and their surroundings, and relating the set of individual actors and their social actions to their surroundings.

Social actors select and construct the appropriate contexts for their social actions. Against this background, social context is regarded as given. ${ }^{1}$ Context is also a constitutive part of the research paradigm of speech act theory. Just consider felicity conditions as social and linguistic context categories (Austin, 1975; Searle, 1969), and language games where language is conceived as a form of action and as a form of life (Wittgenstein, 1958). The impact of social context on language, language use and linguistic performance is also of importance in the field of sociolinguistics. For example, code-switching is anchored to the differentiation between the value-free conceptions of bi- and multilingualism and their value-loaded high- and low-variety counterparts of diglossia, and to different styles and registers (Fasold, 1990; Holmes, 1992; Wardhaugh, 1993). Social context is not only conceived of as presupposed and given, but also as interactionally organized, which is reflected in the research paradigms of conversation analysis (Heritage,1984; Schegloff, 1991), ethnomethodology (Garfinkel, 1994) and ethnography of speaking (Saville-Troike, 1989). Here, context is generally differentiated with regard to a spatio-oriented frame of reference and a socio-oriented frame of reference, namely the immediate physical surroundings,

\footnotetext{
1 This is reflected in the presuppositional approach to context which employs terms like common ground, background information or background assumptions (Penco, 1999; Stalnaker, 1999; van Dijk, 1977).
} 
which are assigned the status of common ground to which the co-participants anchor their social actions (Akman, 2000). Social action is therefore dependent on context and interacts with context. In addition to the social or external context, utterances in the field of conversation analysis and communicative acts in the field of sociopragmatics represent another type of context: linguistic context. Here, the language produced (formulated) and interpreted (decoded) by the co-participants is assigned a dual function. On the one hand, it invokes linguistic context by constructing it itself; on the other hand, its sole production and interpretation provide the context for subsequent talk and recovery of intended meaning (Akman and Alpaslan, 1999). Accordingly, the act of speaking and interpreting builds contexts and at the same time constrains the building of contexts. To employ Heritage's (1984, p. 242) terminology, the production of talk is doubly contextual. An utterance relies upon the existing context for its production and interpretation, and it is, in its own right, an event that shapes a new context for the action that will follow.

Contrary to a conception of language as an autonomous system (as, for instance, is the case for formal linguistics), ethnographic, ethnomethodological and conversation-analytic frameworks assign language the status of a sociocultural and context-dependent means of communication. In these dynamic frames of reference, social contexts are jointly constructed and negotiated. Analogously to the ethnomethodological conception of social context, more recent approaches to context are also based on a dynamic conception of context and explicitly account for the accommodation of communicative meaning which is constructed and negotiated in communication (Clark, 1996; Duranti and Goodwin, 1991; Thomason, 1992). Thus, a dynamic approach to communication in context is based on the premises that social action and language use (i) are embedded in a context of situation, (ii) constitute practical action and (iii) are a socially situated cultural form. So, where do linguistic and social contexts meet, and where do they depart?

\section{Linguistic context}

Language use is necessarily anchored to a speech situation, co-participants and language, and is therefore embedded in the immediate physical surroundings which are available in local linguistic context(s) and in more remote linguistic context(s), and in immediate extra-linguistic context(s) and in more remote extra-linguistic context(s). Depending on the theoretical frameworks, the linguistic context of social action comprises a communicative contribution's adjacent communicative contributions, a speech act's adjacent speech acts, an utterance's adjacent utterances or a turn's adjacent turns. Yet, is it only the prior contribution that constitutes linguistic context or is it the prior and the upcoming contributions which constitute the linguistic context of a current contribution? And in what way are these linguistic strings related?

In discourse analysis, it is generally assumed that a current contribution requires a prior contribution in order to account for anaphora resolution and for the retrieval of other contextual information. In a dynamic outlook on communication, however, 
a current contribution is not only anchored to a prior contribution, but also constrains the form and function of upcoming talk. For instance, an information question generally requires a dovetailed answer which provides the requested information. An information question only allows a question as an upcoming contribution if it functions as a request for a specific type of information referred to in the prior question. Against this background, the delimitation of linguistic context to a contribution's directly or immediately adjacent contributions seems too narrow. Rather, linguistic context is conceived as delimited by the contextual constraints and requirements of a communicative genre (Luckmann, 1995), which entails the notions of macro speech act (van Dijk, 1977), text type (De Beaugrande and Dressler, 1981) and communicative project (Linell, 1998). These macro categories are hierarchically structured and sequentially organized, and they subcategorize into pre-, core-, side- and closing sequences. In a dynamic outlook on communication, linguistic context can be decontextualized at a local level and recontextualized at a global level. As regards the former, linguistic context is decontextualized in a negotiation of meaning sequence in order to repair some misunderstanding. As regards the latter, the reproduction of local meaning, as is the case with the phenomenon of reported speech, for example, is recontextualized at a global level by adding further contextual information. Thus, there is intradiscursive recontextualization anchored to local decontextualization and interdiscursive recontextualization anchored to global recontextualization.

Similar to a communicative contribution and a speech act, the macro categories of a communicative genre, a macro speech act and a text type are defined by intentionality of action and responsibility for possible consequences. Luckmann (1995, p. 177) defines the macro category of a communicative genre as follows:

Communicative genres operate on a level between the socially constructed and transmitted codes of "natural" languages and the reciprocal adjustment of perspectives, which is a presupposition for human communicative interaction. They are a universal formative element of human communication. [...] Human communicative acts are predefined and thereby to a certain extent predetermined by an existing social code of communication. This holds for both the "inner" core of that code, the phonological, morphological, semantic and syntactic structure of the language, as well as for its "external" stratification in styles, registers, sociolects and dialects. In addition, communicative acts are predefined and predetermined by explicit and implicit rules and regulations of the use of language, e.g. by forms of communicative etiquette.

A necessary condition for the differentiation of context into linguistic context and social context is a conception of language as a vehicle for communication and conversational interaction. Against this background, linguistic context is intrinsically linked to social context which is also clearly shown in the definitions of the macro category of a communicative genre which is based on the social-context concept of convention. The micro-macro link made explicit in the definition of a communicative genre is also reflected in the distinction between local social and global social contexts. But what is a social context; or more precisely, what are its constituents? 


\section{Social context}

The research paradigm of ethnomethodology represents a micro sociological perspective par excellence. It is based on the premise that indexicality of social action is key and it focuses on the domain of intersubjectivity. It examines the questions of how separate individuals are able to know or act within a common world and of how members negotiate or achieve a common context. ${ }^{2}$ This common context is synonymous with social context, which, like linguistic context, classifies into a local (or micro) social context and a global (or macro) social context (Schegloff, 1987). Social contexts are further distinguished with regard to a number of intermediate layers, such as meso social context regarding the delimiting frame of a particular speech event or the more global institutional context (Boden and Zimmerman, 1993; Drew and Heritage, 1992; Sarangi and Slembrouck, 1996).

Social context is often considered to comprise the context of a speech event and is defined by the deduction of linguistic context and cognitive context from a holistic conception of context as comprising all of the constitutive parts of a speech event. Constituents of social context are, for instance, co-participants, ${ }^{3}$ micro context (that is, the immediate concrete, physical surroundings including time and location), and the macro contextual institutional and non-institutional domains. Frequently, language use in social contexts has been allocated to the domains of linguistic performance or parole which have been assigned the status of an individual and momentary product. This has been denied by Recanati (1998, p. 133): 'But to this we can now reply that there exist, "deposited in each brain", rules for the game of speech, common to all and existing independently of the will of the individual.' This is also manifest in the prototypical micro contextual phenomenon of deixis and deictic expressions (i.e. temporal deixis, local deixis, participant deixis, discourse deixis and social deixis). Contrary to anaphora resolution, which requires linguistic contexts, discourse deixis is informed both by linguistic and social contexts.

As regards the status of the co-participants, the categories of speaker, hearer and audience denote interactional categories. The speaker is the one who produces (encodes) communicative contributions. The hearer is the one who interprets (decodes) communicative contributions. The audience, on the other hand, interprets/ decodes the communicative contributions. In an actual speech situation, however, interactional categories do more than simply produce and interpret communicative contributions. In a social context, they subcategorize into social roles and their gendered and ethnic identities. In institutional communication the co-participants' institutional roles embody institutional power which manifests itself in their corresponding rights and obligations. True, the concepts of a speaker, a hearer and an audience are, from a language-production and language-interpretation viewpoint, fairly straightforward. However, if these interactional roles are instantiated in social

\footnotetext{
2 See Davidson (2001) for a rewarding examination of the nature and status of knowledge of our own minds, knowledge of the contents of other minds, and knowledge of the shared environment.

3 Co-participants further subdivide (Goffman, 1974; Levinson, 1988) into the domains of speaker, hearer and audience, and their respective subcategories such as author, principal, strategist, emitter, addressee, target, etc. and further discern with regard to their status (ratified and unratified).
} 
contexts, they become more complex. For this reason, speakers, hearers and audience are represented by multiple social roles. As for their status in discourse, one would like to represent multiple discourse identities.

So far, the examination of the constitutive parts of a speech situation has focussed on its unmarked form, namely face-to-face interaction. The complexity of interacting and interdependent roles increases in mediated discourse, such as mediation talk or media communication, where different frames and different levels of talk interact. In media communication, we have a first-frame interaction and the corresponding first-frame roles, and then we have a second-frame interaction, for which the firstframe interaction serves as input (Fetzer, 2000). Analogously to the relational conception of context, we do not have separate, but, rather, interacting frames. This also holds for telephone conversations, where there is still a direct interaction and a direct negotiation of meaning. By contrast, in a written speech situation one has delayed-response and, thus, no direct negotiation of meaning. To conclude, the social context of a speech situation is multifaceted and hence extremely complex, and it is, in practice, refined by a sociocultural context. But what exactly are sociocultural contexts? ${ }^{4}$

\section{Sociocultural context}

Social context is frequently used synonymously with extra-linguistic context which comprises the co-participants, their physical and psychological dispositions and the specific knowledge or assumptions about the persons involved, the knowledge of the language, routines and activity-types (Levinson, 1992), their communicative intentions and communicative goals, and general background knowledge. Of course, the immediate extra-linguistic context is embedded in more remote extra-linguistic contexts, such as particular organizational contexts and other socio-historically constituted contexts of institutions and (sub)cultures. The synonymous use of extra-linguistic context and social context is, however, an oversimplification as research in sociolinguistics, anthropology and cultural studies has informed us. Rather, social context subcategorizes into different types of sociocultural context which are defined by a particular perspective on social context in general. Thus, social context and all of its constituents can be conceived as an unmarked type of context or as a default context, and sociocultural context can be conceived as a marked type of context in which particular variables, such as time or location, are interpreted in a particular mode. For instance, the distinction between monochronic time and polychronic time is based on

\footnotetext{
${ }^{4}$ Before we delve into this question, we would like to make a passing remark re validity basis of speech - a crucial component of social context. Habermas (1998) believes that communication presupposes a norm which demands intelligibility, truth, warrant and sincerity, encapsulated by the ratification of validity claims (Fetzer, 1999). Defined by this norm is an ideal pragmatic situation in which communication cannot be distorted by social factors. Habermas's work is valuable because it emphasizes factors like the knowledge of speaker's personal history or familiarity with the culturally specific contexts of a discourse. In a nutshell, Habermas sees language as a specific medium for coming to an understanding on the sociocultural plane.
} 
the differentiation between a linear, tangible and divisible conception of time, where events are scheduled one at a time and where this schedule takes precedence over interpersonal relationships. Polychronic time, by contrast, is characterized by things occurring simultaneously. Here, interpersonal relationships take precedence over a task-oriented outlook on communication (Hall and Hall, 1989). In other words, culture provides us with a filter mechanism which allows us to interpret social context in accordance with particular sociocultural-context constraints and requirements.

The aforementioned view is also reflected in Hymes's (1974) notion of a speaking grid anchored to the components of situation (the physical setting and the psychological scene), participants (speaker, hearer and audience, and their status in the participation framework), ends (the goal and the purpose of the speech event from a sociocultural viewpoint), act sequence (how something is said as regards message form and what is said as regards message content), key (mock or serious), instrumentalities (channels - spoken, written, e-mail-and forms - vernacular, dialect, standard - of speech), norms (of interaction and interpretation) and genres. Hymes's speaking grid and his notion of communicative competence has been refined by Gumperz's $(1977,1992)$ conception of conversational inference, which is related to his notion of contextualization cue. The latter is explained by Saville-Troike (1989, p. 131) as follows:

Gumperz builds on this [the extent to which speakers must share experience to successfully develop conversational exchanges of any length and depth-AF \& VA] in proposing the outline of a theory of how social knowledge is stored in the mind, retrieved from memory, and integrated with grammatical knowledge in the act of conversing. Conversational inference is "the "situated" or contextbound process of interpretation, by means of which participants in a conversation assess others' intentions, and on which they base their responses' (Gumperz, 1977, p. 191).

Because of its cultural base, the 'meaning' that emerges in a conversation is likely to be different for participants if they are not members of the same speech community.

It is of relevance that these investigations do not refer to social context but rather to sociocultural context. This is due to the fact that language is seen as a socially situated cultural form and, therefore, as a specification of the more generally conceived social and linguistic contexts. Yet linguistic context is not only conceived of from a local or micro perspective. Global or macro linguistic context manifests itself in the above-explicated category of a communicative genre. The research domain of language-as-social-semiotic, such as the ethnography of speaking, recognizes the necessity of analyzing the linguistic code itself. However, there are also numerous investigations which focus on the cognitive processes of speakers and hearers that are required for the performance of communicative acts in a sociocultural setting (Gumperz, 1992; Saville-Troike, 1989).

Cognitive context denotes a set of premises and cognitive environment denotes a set of facts, namely, true or possibly true mental representations. Constitutive 
elements of cognitive context in a relevance-theoretic frame of reference (Sperber and Wilson, 1996) are mental representations, propositions, contextual assumptions which may vary in strength, and factual assumptions. Assumptions are read, written and deleted. In the meantime, contextual implications are raised in strength, lowered in strength or erased from memory. Since cognitive contexts are anchored to an individual but are also required for a cognitively based outlook on communication, they must contain assumptions about mutual cognitive environments. Intention is also anchored to cognitive context. Following Sperber and Wilson, we claim that intention is a mental representation capable of being realized in the form of actions. ${ }^{5}$ It differentiates into an informative intention, namely, the intention to make manifest or more manifest to the audience a set of assumptions, and the second-order informative intention, the communicative intention, viz. the intention to have the informative intention recognized and to make it mutually manifest to the audience and the communicator that the communicator has this informative intention (Sperber and Wilson, 1996, pp. 54-64). Yet, intention is not only seen from a cognitive perspective. Intention is also considered to be a constitutive element of social action and action theory. And it is at the locus of intentionality where linguistic, social, sociocultural and cognitive contexts meet. Against this background, social actors select and construct the appropriate contexts for their social actions. But what exactly are appropriate contexts?

\section{Appropriate contexts}

The concept of appropriateness supplements and refines the notion of contextdependent pragmatic meaning by social-context and sociocultural context perspectives, where pragmatic meaning is not only calculated with regard to its illocutionary goal and force, but also with regard to the connectedness between pragmatic meaning, co-participants, their social status, interpersonal relationship and communicative setting. Thus, appropriateness is not only conceived of as a context-dependent, but also as a sociocultural notion. To quote Saville-Troike (1989, p. 22): 'Shared cultural knowledge is essential to explain the shared presuppositions and judgements of truth value which are the essential undergirdings of language structures, as well as of contextually appropriate usage and interpretation.' Against this background, appropriateness is anchored to the dyad of (minimally) a speaker and a hearer seen from both I-we (Searle, 1995) and I-thou perspectives (Brandom, 1994), and to the immediate and remote linguistic, sociocultural and social contexts.

If we adopt Linell's (1998) distinction between monologue and dialogue, appropriateness represents a dialogical concept. That is, the appropriateness of dialogue cannot be calculated in the framework of individual contributions only. Rather, it

\footnotetext{
5 Compare this view with Davidson's (2001, p. 125): 'Evaluative attitudes, including desires, intentions, moral convictions, views about duties, and obligations, are [...] propositional attitudes. [...] Wants and desires are directed to propositional contents. [...] Someone who intends to go to the opera intends to make it the case that he is at the opera.'
} 
must be conceived as a relational concept which connects the domain of linguisticsboth linguistic competence and linguistic performance-with the domain of naturallanguage communication-sociocultural competence and sociocultural performance. Moreover, appropriateness is a social concept whose frame of reference goes beyond the individual contribution. That is, both the production and interpretation of an utterance require linguistic, sociocultural and social contexts, which can be spelled out explicitly in the linguistic surface or which can be represented implicitly and assigned a presuppositional status. As a consequence of this, the appropriateness of dialogue is interdependent on a dialogue's textual domain (its linguistic representation), interpersonal domain (the linguistic representation of references to the coparticipants regarding their social and interactional statuses, and their facewants and face-needs), and interactional domain (the linguistic representation of adjacency relations regarding the dialogue as such and the coparticipants' employment of the turn-taking system). But dialogue does not only account for the individual co-participants, but also for their connections and therefore for intersubjectivity and reciprocity. Thus Brandom (1994, p. 170): 'Putting a claim forward as true is putting it forward as one that it is appropriate for others to take as true, that is to endorse themselves.'

This extension of frame is also reflected in Goody's conception of appropriate role behaviour (Goody, 1995, p. 14): 'A definition of appropriate role behaviour includes both the proper behaviour of ego, and the proper reciprocal responses of the role partner. In this way it makes AIP [anticipatory interactive planning-AF \& VA] more powerful, since acting in a clearly defined role makes the behaviour of both members of the dyad more predictable.' So, natural-language communication does not only consist of ego putting a claim forward and other taking it as true, but also of ego's and other's performances in accordance with clearly defined roles.

\section{This issue}

Papers in this special issue were submitted upon invitation. They were then evaluated and revised, following the usual reviewing procedure of Language \& Communication. While we have tried hard to summon the best exemplary work treating contexts of social action, the coverage provided is surely limited in scope. Our expectation is that this special issue will encourage other researchers working in this area to contribute to the future issues of $L \& C$.

Following this introduction, the special issue includes three "theory" papers. These are (in order of appearance) contributed by Jeff Malpas, Marina Sbisa and Roy Dilley.

In "The weave of meaning: holism and contextuality," Malpas offers a panoramic vision of context from a philosophical perspective. While he lucidly diagnoses this powerful notion's resistance to attempts to render it exact, he also underlines its importance in discussions of natural language semantics. For him, context brings about a certain holistic stance: contextual meaning argues for holism in meaningthe extent to which various aspects of meaning depend on each other. But the holism 
that he speaks about is not somehow unconstrained; it is attained only in view of certain settings (locales).

Sbisa's "speech acts in context" is a broad survey of the use of context in clarifying the meaning of speech acts. She argues for unbiased yet circumscribed contexts for situating speech acts and evaluating them. In her vision, there is a close partnership between speech act and context, and only through a close examination of this association it becomes plausible to regard a particular speech act as genuine social action.

Dilley's "The problem of context in social and cultural anthropology" basically carries the message of a recent book he has edited (The Problem of Context). This paper studies the building of context as a key step in anthropological studies. To contextualize, according to Dilley, is to draw a line. Such an act involves the inclusion of what is deemed to be germane and the exclusion of what is reckoned as impertinent. The author proposes that anthropologists must take heed of this point carefully, for it evidently hints at a power conflict. They, as commentators, must be prepared to allow for contexts based upon the bodies of knowledge of natives that they are observing. This view of agents situated in and relativized to a social/historical interval is indispensable. Anthropologists cannot simply assume that there is an objective stance that banishes context-sensitivity.

Three "practice" papers conclude our special. These are (in order of appearance) written by Dick Janney, Paul Drew and Ruth Wodak.

In "Cotext as context: vague answers in court," Janney examines the most salient contributor to a context, viz. cotext. As Lyons (1995, p. 258) argued, text and context should be considered as complementing each other: "Texts are constituents of the contexts in which they are produced; and contexts are created, and continually transformed and refashioned, by the texts that speakers and writers produce in particular situations." It is then only common sense to predict that the cotext (i.e. the relevant or immediate surrounding text) bears upon the question of contextual reasoning and context renewal. Janney's interest lies in the relevance of cotext to the interpretive practices in courtroom testimony and discourse.

Drew's "Out of context: an intersection between domestic life and the workplace, as contexts for (business) talk" looks at the interface between household life and the workplace. As a bona fide specimen of conversation-analytic work, it provides considerable amount of data to explore the connections between talk and social context. Although limited to a particular example (a man at work being phoned by his wife), Drew's insights are fruitful in that they point out to the interaction of context and speaker identity in the detailed unfolding of a talk.

Finally, Wodak gives an insightful analysis in "Friend or foe: the defamation of political opponents or legitimate and necessary criticism? Reflections on recent political discourse in Austria" of how contexts of political crusades might camouflage hideous anti-Semitic arguments and prejudices. ${ }^{6}$ Taking her point of departure as the sensitive domain of the Holocaust, she scrutinizes, with painstaking analysis,

\footnotetext{
${ }^{6}$ The reader is referred to Gilman and Zipes (1997) for an impressive historical account of related (and numerous other) matters.
} 
certain politicians' discursive strategies and vicious argumentation techniques during their recent election campaigns in Austria.

\section{Conclusion}

The construct of social context is based on the premise of agents who share a common linguistic code and employ it as a means of communication. It is at the interface of any sociocultural linguistic performance where social, linguistic and cognitive contexts meet. It is a necessary condition for the successful performance of a communicative act to be tied to linguistic, cultural and cognitive surroundings. We are confident that papers in this issue of $L \& C$ demonstrate the importance and fertility of research into the theoretical and practical aspects of such contexts of social action.

\section{Acknowledgement}

We are grateful to Talbot Taylor for encouragement and helpful advice.

\section{References}

Akman, V., 2000. Rethinking context as a social construct. Journal of Pragmatics 32 (6), 743-759.

Akman, V., Alpaslan, F.N., 1999. Strawson on intended meaning and context. In: Bouquet, P., Serafini, L., Brézillon, P., Benerecetti, M., Castellani, F. (Eds.), Modeling and Using Context (Proceedings of Context '99). Springer, Berlin, pp. 1-14.

Asher, R.E. (Ed.), 1994. The Encyclopaedia of Language and Linguistics. Pergamon Press, Oxford.

Austin, J.L., 1975. How to Do Things with Words (The William James Lectures delivered at Harvard University in 1955). Harvard University Press, Cambridge, Massachusetts. (Urmson, J.O., Sbisa, M., Eds.; second edition).

Boden, D., Zimmerman, D. (Eds.), 1993. Talk and Social Structure: Studies in Ethnomethodology and Conversation Analysis. Polity Press, Cambridge.

Brandom, R.B., 1994. Making It Explicit: Reasoning, Representing, and Discursive Commitment. Harvard University Press, Cambridge, Massachusetts.

Clark, H.H., 1996. Using Language. Cambridge University Press, Cambridge.

Davidson, D., 2001. Subjective, Intersubjective, Objective. Clarendon Press, Oxford.

De Beaugrande, R., Dressler, W., 1981. Einführung in die Textlinguistik. Niemeyer, Tübingen.

Drew, P., Heritage, J. (Eds.), 1992. Talk at Work: Interaction in Institutional Settings. Cambridge University Press, Cambridge.

Duranti, A., Goodwin, C. (Eds.), 1991. Rethinking Context: Language as an Interactive Phenomenon. Cambridge University Press, Cambridge.

Fasold, R., 1990. The Sociolinguistics of Language. Blackwell, Oxford.

Fetzer, A., 2000. Negotiating validity claims in political interviews. Text 20 (4), 1-46.

Fetzer, A., 1999. Validity claims: assigning contextual information. In: Wilson, T.D., Allen, D. (Eds.), Information Seeking in Context (Proceedings of ISIC'98). Taylor Graham, London, pp. 494-505.

Garfinkel, H., 1994. Studies in Ethnomethodology. Polity Press, Cambridge.

Gilman, S.L., Zipes, J. (Eds.), 1997. Yale Companion to Jewish Writing and Thought in German Culture, 1096-1996. Yale University Press, New Haven, Connecticut. 
Goffman, E., 1974. Frame Analysis. Northeastern University Press, Boston.

Goody, E.N., 1995. Introduction: some implications of a social origin of intelligence. In: Goody, E.N. (Ed.), Social Intelligence and Interaction: Expressions and Implications of the Social Bias in Human Intelligence. Cambridge University Press, Cambridge, pp. 1-36.

Gumperz, J.J., 1977. Sociocultural knowledge in conversational inference. In: Saville-Troike, M. (Ed.), Linguistics and Anthropology (28th Annual Round Table Monograph Series on Languages and Linguistics). Georgetown University Press, Washington, DC, pp. 191-211.

Gumperz, J.J., 1992. Contextualization and understanding. In: Duranti, A., Goodwin, C. (Eds.), Rethinking Context: Language as an Interactive Phenomenon. Cambridge University Press, Cambridge, pp. 229-252.

Habermas, J., 1998. On the Pragmatics of Communication. MIT Press, Cambridge, Massachusetts. (Essays translated from German; Cooke, M., Ed.).

Hall, E.T., Hall, M.R., 1989. Understanding Cultural Differences: Germans, French and Americans. Intercultural Press, Yarmouth, Maine.

Heritage, J., 1984. Garfinkel and Ethnomethodology. Polity Press, Cambridge.

Holmes, J., 1992. An Introduction to Sociolinguistics. Longman, London.

Hymes, D., 1974. Foundations in Sociolinguistics: An Ethnographic Approach. University of Pennsylvania Press, Philadelphia.

Levinson, S.C., 1992. Activity types and language. In: Drew, P., Heritage, J. (Eds.), Talk at Work: Interaction in Institutional Settings. Cambridge University Press, Cambridge, pp. 66-100.

Levinson, S.C., 1988. Putting linguistics on a proper footing: explorations in Goffman's concepts of participation. In: Drew, P., Wootton, A. (Eds.), Erving Goffman: Exploring the Interaction Order. Polity Press, Cambridge, pp. 161-227.

Linell, P., 1998. Approaching Dialogue. John Benjamins, Amsterdam.

Luckmann, T., 1995. Interaction planning and intersubjective adjustment of perspectives by communicative genres. In: Goody, E.N. (Ed.), Social Intelligence and Interaction: Expressions and Implications of the Social Bias in Human Intelligence. Cambridge University Press, Cambridge, pp. 175-188.

Lyons, J., 1995. Linguistic Semantics: An Introduction. Cambridge University Press, Cambridge.

Penco, C., 1999. Objective and cognitive context. In: Bouquet, P., Serafini, L., Brézillon, P., Benerecetti, M., Castellani, F. (Eds.), Modeling and Using Context (Proceedings of Context '99). Springer, Berlin, pp. 270-283.

Recanati, F., 1998. Meaning and force: introduction. In: Kasher, A. (Ed.), Pragmatics: Critical Concepts. Routledge, London, pp. 126-154.

Sarangi, S., Slembrouck, S., 1996. Language, Bureaucracy and Social Control. Longman, London.

Saville-Troike, M., 1989. The Ethnography of Communication. Blackwell, Oxford.

Schegloff, E., 1991. In another context. In: Duranti, A., Goodwin, C. (Eds.), Rethinking Context: Language as an Interactive Phenomenon. Cambridge University Press, Cambridge, pp. 191-228.

Schegloff, E.A., 1987. Between macro and micro: contexts and other connections. In: Alexander, J., Giesen, B., Munch, R., Smelser, N. (Eds.), The Micro-Macro Link. University of California Press, Los Angeles, pp. 207-234.

Searle, J.R., 1995. The Construction of Social Reality. Free Press, New York.

Searle, J.R., 1969. Speech Acts: An Essay in the Philosophy of Language. Cambridge University Press, Cambridge.

Sperber, D., Wilson, D., 1996. Relevance: Communication and Cognition. Blackwell, Oxford.

Stalnaker, R.C., 1999. Context and Content: Essays on Intentionality in Speech and Thought. Oxford University Press, Oxford.

Thomason, R.H., 1992. Accommodation, meaning, and implicature: interdisciplinary foundations for pragmatics. In: Cohen, P.R., Morgan, J., Pollack, M.E. (Eds.), Intentions in Communication. MIT Press, Cambridge, Massachusetts, pp. 325-363.

Van Dijk, T., 1977. Text and Context. Longman, London.

Wardhaugh, R., 1993. An Introduction to Sociolinguistics. Blackwell, Oxford.

Wittgenstein, L., 1958. Philosophical Investigations. Blackwell, Oxford. [Anscombe, G.E.M. (trans.)]. 
Anita Fetzer

Institut für Linguistik: Anglistik, Universität Stuttgart, Keplerstraße 17 D-70174 Stuttgart, Germany E-mail address: anita@ifla.uni-stuttgart.de $U R L:$ http://ifla.uni-stuttgart.de/fetzer/index.html

\author{
Varol Akman \\ Department of Computer Engineering, Bilkent University Bilkent \\ Ankara 06533, Turkey \\ E-mail address: akman@cs.bilkent.edu.tr \\ $U R L:$ http://www.cs.bilkent.edu.tr/ akman
}

